\title{
Non-linear models for Rotor-AMB system drop
}

\author{
C. Jarroux ${ }^{1,2}$, R. Dufour ${ }^{1}$, J. Mahfoud ${ }^{1}$, B. Defoy ${ }^{2}$, T. Alban ${ }^{2}$ and A. Delgado ${ }^{3}$ \\ ${ }^{1}$ Université de Lyon, CNRS, INSA-Lyon, LaMCoS UMR5259, 69621 Villeurbanne, France \\ ${ }^{2}$ Thermodyn, GE Oil and Gas, 71200 Le Creusot, France \\ ${ }^{3}$ GE Global Research Center, NY 12309 Niskayuna, USA
}

\begin{abstract}
The proposed investigation deals with the assessment of several models for the prediction of the dynamic behaviour of turbomachinery supported by Active Magnetic Bearing when drop events occur leading the rotor to drop onto its Touch-Down Bearings (TDBs), which are emergency rolling element bearings in series with a ribbon damper. The rotor exhibits a non-linear transient motion where the amount of damping provided by the ribbon damper is a key parameter for avoiding dangerous behaviours. The ribbon damper is modelled successively with the Masing, the generalized Dahl and the Kelvin-Voigt models. Usually, the TDB is modelled as a viscous damper, used here as reference for comparisons. The originality of this work lies in the new modellings of that ribbon, based on dry-friction theory, where parameters are benchmarked to experimental results. The three models presented are successively integrated in the TDB model followed by rotor drop simulations. Comparisons are done in terms of rotor drop dynamics and show that the transient responses predicted with the Masing and generalized Dahl models are similar.
\end{abstract}

\section{Introduction}

Rotating machines supported by Active Magnetic Bearings (AMBs) lead to frictionless system with reduced energy losses. However, in some particular events, the AMB power supply may fail leading to a non-linear transient behaviour of the horizontal rotor mainly subject mass unbalance, contact and gravity forces. The resulting dynamic behaviour has been studied over the last twenty years [1-6]. The rotor, forced by gravity, drops onto its Touch-Down Bearings (TDBs), ball-bearings in this case, providing a back-up pivot linkage and protecting the AMBs. The rotor can follow several types of trajectory after impact. Its dynamic behaviour depends mostly on the amount of damping in the system, mainly provided by the ribbon damper, a corrugated steel foil fitted between the outer-race of the TDB and the casing $[5,6]$, see figure 1 .

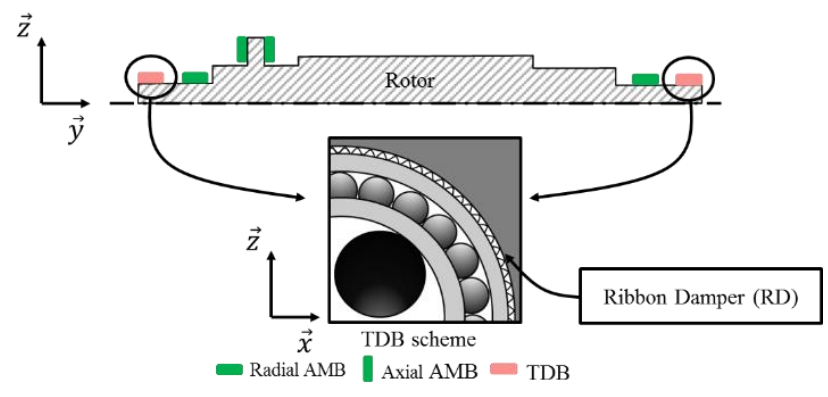

Fig. 1. Schematic position of AMBs and TDBs
With the purpose of enhancing rotor drop dynamic predictions, measured force-deflection loops were performed to evaluate the dynamic behaviour of the ribbon damper. The latter is related to dry-friction phenomenon rather than viscous damping, as it is usually assumed. The first part of this work describes the test-rig and experimental results. In the following section, the Masing and generalized Dahl models, both dry-friction models, are described and adjusted to fit experimental results. The Kelvin-Voigt model, considered as the reference, is used with provided data. Then, an eight degrees of freedom (DoF) model is established, comprising 3-DoF rigid rotor and 5-DoF TDB models. Finally, the influence of the three TDB models on the rotor drop dynamics is investigated.

\section{Experiments}

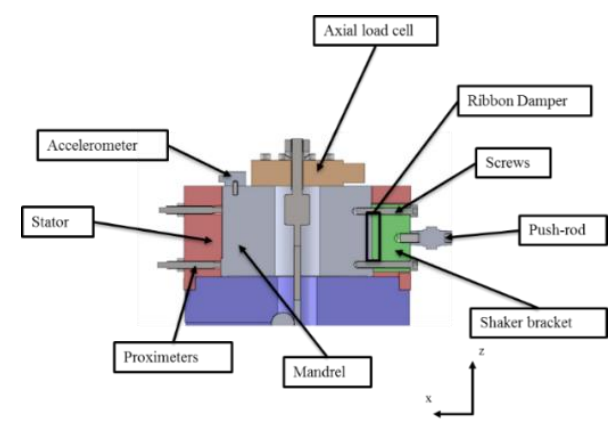

Fig. 2. Vertical cross-section of the ribbon damper test-rig 


\subsection{Test-rig}

The objective of the experiments was to measure the restoring forces generated by the preloaded corrugated foil (ribbon damper). Figure 2 shows a cross-section of the test-rig comprising a stationary housing and a movable mandrel connected to two electro-hydraulic shakers $(90$ deg. apart) via threaded rods instrumented with load cells. The ribbon is positioned between the housing and the mandrel. The housing includes Eddy-current sensors to measure relative motion of the mandrel while an accelerometer atop the mandrel measures acceleration.

The mandrel was excited with single frequency excitations along a single axis ranging from $20 \mathrm{~Hz}$ to $200 \mathrm{~Hz}$. Once the mandrel reached steady state, $0.5 \mathrm{~s}$ of signal was recorded using a sampling frequency of $8192 \mathrm{~Hz}$. The ribbon reaction force is obtained by subtracting the mandrel inertial force from the shaker input force. The experimental results indicate the restoring force is a hysteresis force-deflection loop quasi-symmetric regarding the zero origin, which denotes that the compression and traction phases are rather similar. In addition, sharp corners at the change of the velocity indicate the dynamic behavior of the ribbon damper should be numerically described by dry-friction models.

\subsection{Dry-friction phenomenon}

The dry-friction damping induces a highly non-linear force which is able to flatten the frequency response of dynamic systems. This peak flattening could be due to the out-ofphase between the friction force and the displacement generated by the stick-slip phenomenon, as explained in [7]. Thus, the damping provided by the ribbon when a rotor drops onto its TDBs would come mainly from the alternation of stick and slip motion of each bump of the ribbon, rubbing along the inner-race and housing surfaces, generating this force-deflection out-of-phase. Two main categories of friction model can be employed: macroslip and microslip models. Macroslip models generate homogenous dynamics meaning that everything slips or everything sticks at the same time, while in the microslip model mixed configurations appear. In the case of the ribbon, the large imposed displacements generate brutal stick-slip transition while this is not absolutely true for small imposed displacements. The next section deals with the description of the ribbon damper models.

\section{Ribbon damper models}

\subsection{The Masing model}

A first approach is the modelling of the ribbon damper with the Masing macroslip model, used in [7] in parallel with a purely elastic restitution force, see equation (1). The contact parameters are assessed thanks to the experimental force-deflection loops, plotted in dotted lines in figure 4 . The stick stiffness $k_{1}$ so-called contact stiffness, corresponds to the scenario where all the ribbon bumps are stuck along the inner-race and housing surfaces, resulting in a high global stiffness. Once the slipping threshold $\mu \mathrm{F}_{\mathrm{N}}$ is reached, all the bumps slip and a change of slope occurs in the hysteretic loop. The resulting stiffness so-called the slipping stiffness $k_{2}$ is much smaller than the contact stiffness. The rubbing device $\mathrm{z}$ records the last position before each change of dynamic behaviour.

$f_{r d}=k_{2} u+f_{n l}\left(z, u, \frac{d u}{d t}\right)$

$\left\{\begin{array}{l}\text { if }\left|k_{1}(u-z)\right|>\mu F_{N} \rightarrow\left\{\begin{array}{l}f_{n l}=\operatorname{sign}\left(\frac{d u}{d t}\right) \mu F_{N} \\ z=u-\operatorname{sign}\left(\frac{d u}{d t}\right) \frac{\mu F_{N}}{k_{1}}\end{array}\right. \\ \text { else }\left\{\begin{array}{l}f_{n l}=\operatorname{sign}\left(\frac{d u}{d t}\right) k_{1}(u-z) \\ z=z\end{array}\right.\end{array}\right.$

\subsection{The generalized Dahl model}

The Dahl model, generalized in [8] and used in [9], is a dry-friction restoring force model, describes by a nonlinear first Order Differential Equation (ODE), detailed in equation (3). The advantage of this model is that it can adopt any shape of loop.

$$
\begin{aligned}
& \frac{d f_{r d}}{d t}=\beta \frac{d u}{d t}\left(h-f_{r d} \operatorname{sign}\left(\frac{d u}{d t}\right)\right) \\
& h=\frac{1}{2}\left(\left(h_{u}+h_{l}\right) \operatorname{sign}\left(\frac{d u}{d t}\right)+\left(h_{u}-h_{l}\right)\right)
\end{aligned}
$$

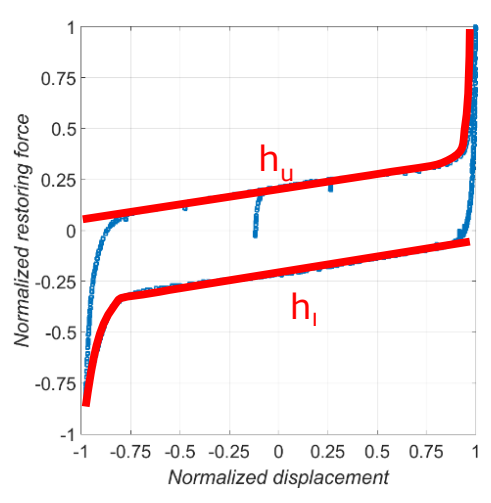

Fig. 3. Ribbon damper experimental results

The $\beta$ term is the only parameter that controls the transition between slip and stick states. Implementing a high value of $\beta$ leads to a "close" macroslip regime. The current envelop $\mathrm{h}$ depends on the sign of the deflection velocity to become either $h_{u}$ or $h_{l}$, which are respectively the upper and the lower asymptotic envelops of the quasi static hysteretic loop plotted in figure 3 . 
As these envelops are curved fitted, one can observe that the hysteretic behavior is characterized by a linear forcedeflection relation from -0.8 to 0.8 , thus indicating the ribbon exhibits a hardening-hardening non-linear behavior for the higher deflections.

\subsection{The Kelvin-Voigt model}

Classically employed in rotor drop dynamic investigations, the linear spring-damper so-called KelvinVoigt model, is described in equation (5). The stiffness is provided by the manufacturer and the damping, measured with logarithmic decrement technique, is set to 0.16 :

$$
f_{r d}=c_{r d} \frac{d u}{d t}+k_{r d} u
$$

The displacements measured with the Eddy-current sensors as well as their time derivatives are the input for the three developed models described by equations (1), (3) and (5). The figure 4 compares the predicted (solid lines) and measured (dotted lines) force-deflection loops. Results are normalized with respect to the ribbon damper clearance. Both dry-friction models provide accurate predictions and are capable of reproducing the a Coulomb model smoothed with the arctangent law, also used in [11]. The TDB angular dynamics is taken into account by an equivalent polar inertia, a resistive torque and the TDB is driven in rotation by the Coulomb interface friction force. The bearing restoring forces are

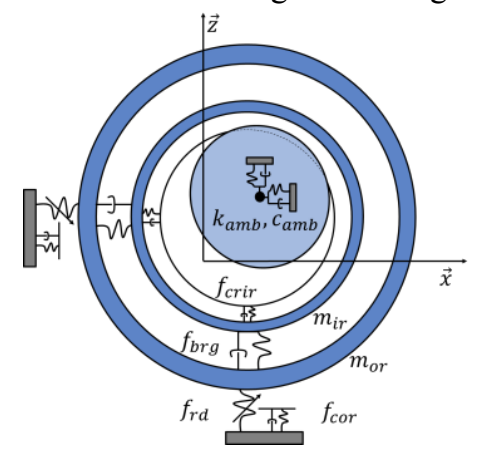

Fig. 5. Schematic presentation of the TDB model

implemented using a quadratic law. The pre-loaded ribbon provides a $f_{r d}$ force. Finally, $f_{\text {cor }}$, called hard-stop, is the contact force between the outer-race and the housing, modeled like $f_{\text {crir. }}$. This force appears when the ribbon is fully crushed.

\subsection{Drop simulations}

The shaft rotates at $6500 \mathrm{rpm}$ and is subject to mass
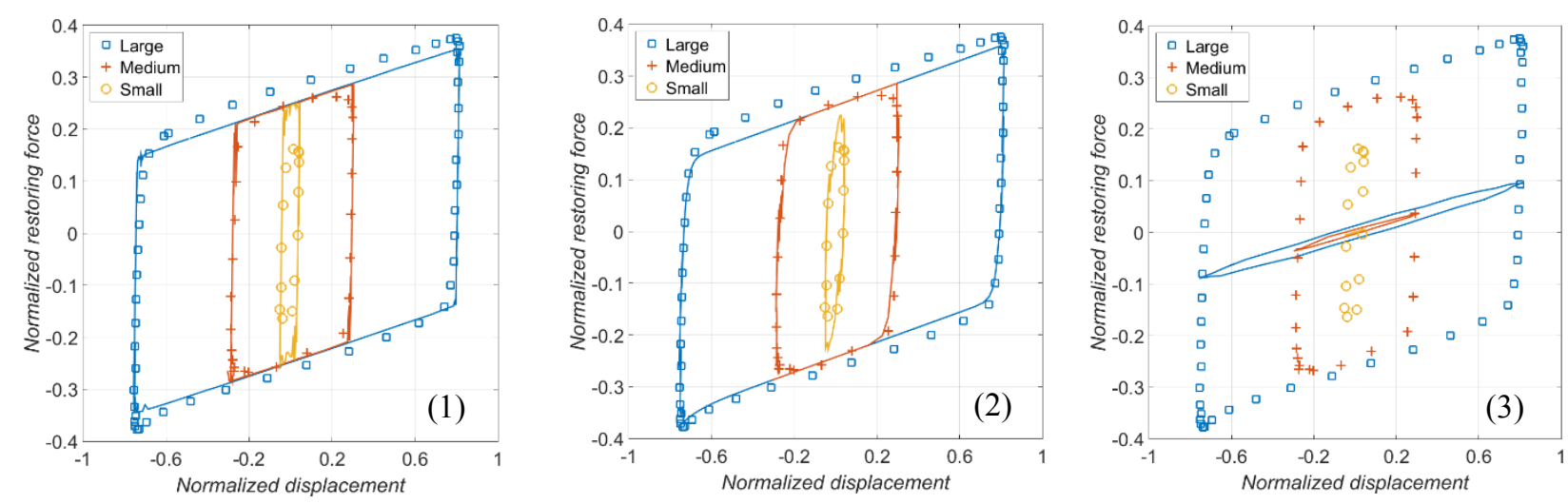

Fig. 4. Force-deflection loop with the Masing model (1), the generalized Dahl model (2) and the Kelvin-Voigt model (3)

phenomenon. On the other hand, the Kelvin-Voigt model, representing only the slip stiffness without including stickslip phenomenon, significantly underpredicts the amount of damping associated with the area defined by the forcedeflection loop (dissipated energy).

\section{Rotor - TDB system}

A classical 5-DoF TDB model [3-6], sketched in figure 5, is used to describe the rotor drop dynamics. The rotor model consists in a rigid rotating mass with 2 lateral DoF and 1 angular DoF. AMBs are considered having constant stiffness $\mathrm{k}_{\mathrm{amb}}$ and damping $\mathrm{c}_{\mathrm{amb}}$ coefficients at a constant rotating speed. The normal contact forces $f_{\text {crir }}$ is modelled with a vibro-impact model based on Hunt and Crossley [10] derived from the Hertz theory for inelastic collisions. The inner-race rotor interface friction force is described by unbalance forces, based on API 617 standard. When the steady-states regime is reached, the AMBs are shut-down and the gravity is applied along the z-axis. The rotor is decelerated by the interface friction force while the TDB accelerates. Time transient simulation of $3 \mathrm{~s}$ are computed using the $5^{\text {th }}$ order Runge-Kutta algorithm with typical time-step for non-linear analysis.

\subsection{Results}

Rotor drop orbits are plotted in figure 6. Results are normalized with respect to the rotor-TDB clearance. Predictions with the dry-friction models are similar. Drop 

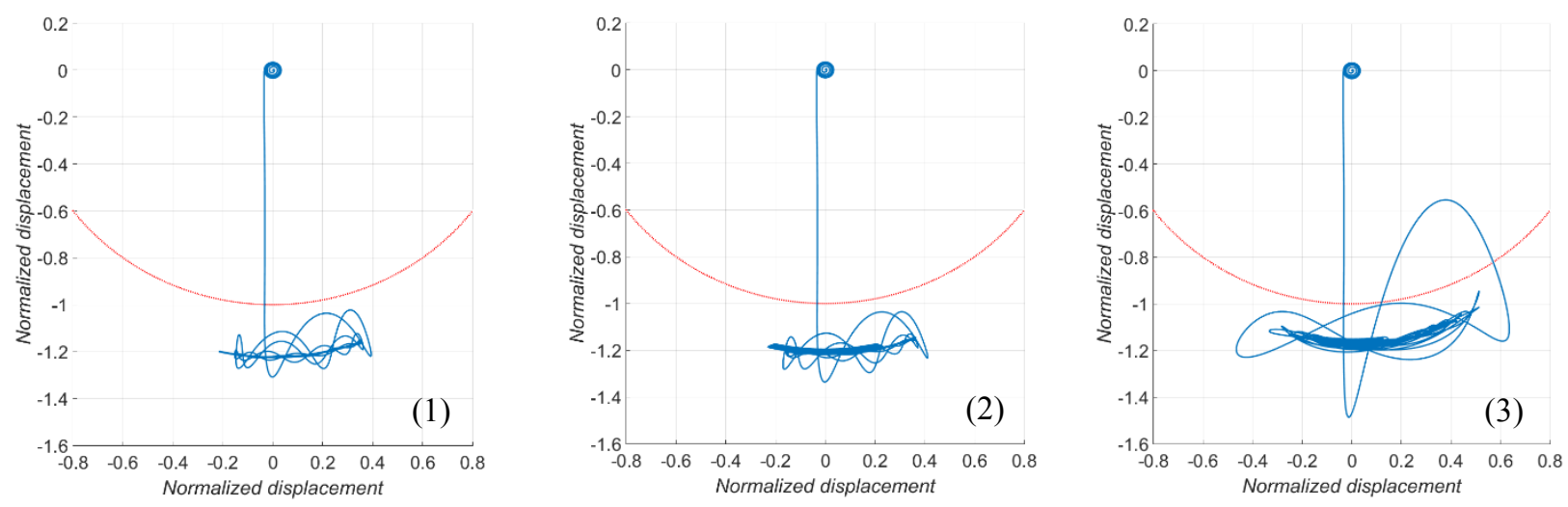

Fig. 6. Rotor orbits with the Masing model (1), the generalized Dahl model (2) and the Kelvin-Voigt model (3)

rebounds are flattened with both the Masing and the generalized Dahl models meaning that the dry-friction generates a considerable amount of damping. In contrast, rotor drop predictions with the Kelvin-Voigt model exhibit rebounds. This is in agreement with observations previously made based on figure 3 . For both the dryfriction model, the rotor drop energy is quite fully absorbed during the first rebound.

\section{Conclusion}

The rotor drop dynamics on three different ribbon damper model was numerically investigated. The reference model was the commonly used Kelvin-Voigt model based on provided data. The two others were the macroslip Masing model and the microslip generalized Dahl model, both based on dry-friction theory and tuned with harmonic tests performed on the ribbon. These last two models were able to reproduce accurately the real dynamic behaviour of the ribbon under harmonic excitations. Both of them provided similar results concerning the rotor drop dynamics. A considerable amount of damping was generated and drop rebounds were flattened. However, rebounds are usually observed in case of rotor drop events as exhibited by the Kelvin-Voigt model predictions. The ribbon damper may have a different dynamic behaviour when subject to shock or harmonic excitations. Shock tests should be performed on that ribbon to validate this assumption.

\section{Acknowledgement}

This work is a part of Clément JARROUX PhD performed with the support of CIFRE funding N0 2013/1376. The authors are grateful to French National Agency ANRT.

\section{References}

[1] A. Gelin, "Etude théorique et expérimentale des comportements dynamiques permanents et transitoires de rotors sur paliers magnétiques," PhD Thesis, INSA de Lyon, Lyon, 1991.

[2] M. O. T. Cole, P. S. Keogh, and C. R. Burrows, "The Dynamic Behavior of a Rolling Element Auxiliary Bearing Following Rotor Impact,"
Journal of Tribology, vol. 124, pp. 406-413, 2001.

[3] G. Sun, "Rotor drop and following thermal growth simulations using detailed auxiliary bearing and damper models," Journal of Sound and Vibration, vol. 289, pp. 334-359, 2006.

[4] J. G. Lee and A. Palazzolo, "Catcher Bearing Life Prediction Using a Rainflow Counting Approach," Journal of Tribology, vol. 134, pp. 031101-031101, 2012.

[5] J. Wilkes, J. Moore, D. Ransom, and G. Vannini, "An improved catcher bearing model and an explanation of the forward whirl / whip phenomenon observed in AMB transient drop experiments," Journal of Engineering for Gas Turbines and Power, vol. 136, pp. 504-515, 2014.

[6] C. Jarroux, R. Dufour, J. Mahfoud, B. Defoy, and T. Alban, "Parametric analysis of a rigid rotor drop onto touchdown bearings," in Colloquium 573 - Coupling and Nonlinear interactions in Rotating Machinery, Lyon, 2015.

[7] B. Al Sayed, E. Chatelet, S. Baguet, and G. Jacquet-Richardet, "Dissipated energy and boundary condition effects associated to dry friction on the dynamics of vibrating structures," Mechanism and Machine Theory, vol. 46, pp. 479-491, 2011.

[8] A. Al Majid and R. Dufour, "Formulation of a Hysteretic Restoring Force Model. Application to Vibration Isolation," Nonlinear Dynamics, vol. 27, pp. 69-85, 2002/01/01 2002.

[9] A. Al Majid and R. Dufour, "Harmonic response of a structure mounted on an isolator modelled with a hysteretic operator: experiments and prediction," Journal of Sound and Vibration, vol. 277, pp. 391-403, 2004.

[10] K. H. Hunt and F. R. E. Crossley, "Coefficient of Restitution Interpreted as Damping in Vibroimpact," Journal of Applied Mechanics, vol. 42, pp. 440-445, 1975.

[11] C. Duran, L. Manin, M. A. Andrianoely, C. Bordegaray, F. Battle, and R. Dufour, "Effect of rotor-stator contact on the mass unbalance response," in IFToMM International Conference on Rotor Dynamics, Milan, 2014. 